\title{
Ways of technical science development in ophthalmology
}

\author{
Irina Vorobyeva ${ }^{1,{ }^{*}}$,Evgeny Delver ${ }^{2}$, and Anatol Belogurov ${ }^{2}$ \\ ${ }^{1}$ Russian Medical Academy of Continuous Professional Education, 2/1, Barrikadnaya Street, \\ Moscow, 125993, Russia \\ ${ }^{2}$ National Medical Research Center of Cardiology, 15A, 3rd Cherepkovskaya Street, Moscow, \\ 121552, Russia
}

\begin{abstract}
In recent years, in the study of diabetic retinopathy the methods of medical engineering and mathematical modeling with the forecast of the course of diseases and given pathogenetic biomarkers in the lacrimal fluid have been widely used. The theoretical aspects of the research are the developed mathematical criteria. The criteria are the mathematical formulas describing objective data of digital ophthalmological equipment and levels of pathogenetic biomarkers in lacrimal fluid and blood serum. The proposed approach allows to create a decision support sys-tem for predicting the development of diabetic retinopathy and diabetic macular edema. The practical application of the mathematical model is reflected in the developed computer program that enables the ophthalmologist to work with the patient's data in automatic mode. The use of the program for the diagnosis and prognosis of a patient with diabetic retinopathy is demonstrated. The work on the proposed method gives the opportunity to reduce the time for assessing the patient's condition and give a timely forecast of the course of the disease, which makes it possible to approach each patient in a personalized way.
\end{abstract}

\section{Introduction}

Digital technologies and methods of mathematical modeling occupy a leading position in engineering practice and other fields of science and technology [1], [2], [3], [4], [5]. Also, mathematical modeling and medical engineering are widely used in medicine, in particular, in ophthalmology [6], [7], [8].

Diabetic macular edema has severe prognosis for vision. Nowadays, there is an opportunity to diagnose diseases early with the detection of pathogenesis biomarkers, which enables us to reach a personalized approach to the treatment of these patients [9].

Research methods: traditional ophthalmology, including fundus photoregistration, optical coherence tomography (OCT), fundus microperimetry MAIA, fluorescent angiography, as well as research of the level of pathogenetic biomarkers in the lacrimal fluid, vascular endothelial growth factor (VEGF-A) and the level of glycated hemoglobin (HbA1c) [10].

* Corresponding author: irina.docent2000@mail.ru 
Neovascularization of the retina can be caused by VEGF-A. Maintaining photoreceptors, endothelial cells, and blood vessels is also a function of VEGF-A. Increased production of VEGF-A is possible in the pericytes of retinal vessels, in the outer and inner nuclear layers of the retina. Basic expression of VEGF-A mRNA is possible in the conjunctiva, eye lens, ciliated body, choroid, and retinal pigment epithelium [11].

In diabetic retinopathy, an increase in the level of VEGF-A in the structures of the eye is noted (more often in the moisture of the anterior chamber, in the vitreous body). There is no correlation between the content of VEGF-A in the eye media in diabetic retinopathy and the concentration in blood plasma, which indicates local synthesis of VEGF-A in diabetic retinopathy. Studies have shown that after vitrectomy a high level of VEGF-A is observed in proliferative membranes, which confirms the important role of angiogenesis [12].

Thus, VEGF is a key regulator of pathological angiogenesis in diabetic retinopathy with proinflammatory activity and it affects the production of chemoattractants, increases the activation of monocytes and leukocytes, and violates the permeability of the hematoretinal barrier [13].

Modern diagnostics of diabetic retinopathy using equipment with the analysis of biomarkers in tears and serum is possible and well-studied. However, it is difficult for a doctor to predict the course of the disease in a particular patient. For an accurate prognosis of the disease, it is necessary to apply mathematical modeling, modern digital technologies and methods of medical engineering [14].

\section{Problem}

In the study of the prognosis of diabetic retinopathy progression and diabetic macular edema two problems were solved:

- a mathematical model for predicting the diabetic retinopathy progression and diabetic macular edema with the creation of mathematical criteria was developed. The criteria describe objective digital data of ophthalmological equipment and the results of biochemical research of biomarkers: the level of vascular endothelial growth factor (VEGFA) in lacrimal fluid and the level of glycated hemoglobin (HbAlc) in serum;

- a computer program based on a mathematical model that enables an ophthalmologist to automatically determine the prognosis of diabetic retinopathy progression and diabetic macular edema was created and implemented.

\section{Materials and methods}

A device called an optical coherence tomograph is used for examining the fundus. It gives the opportunity to determine the structure of the retina. The operation of the device is based on the physical phenomenon of interference. It captures the thickness of the retina in the macular area and transmits it to a computer, which makes it possible to visualize the layers of the retina on the monitor screen [15].

A criterion assessing the effect of the patient's retinal thickness on the prognosis of diabetic retinopathy and diabetic macular edema in type 2 diabetes was developed. The formula is based on the mathematical method of normalization of the studied indicator.

A criterion of the influence of retinal thickness, which is characterized by the severity of the volume of edema in the thickness of the retina in 9 sectors of the macular zone, is proposed: 


$$
R_{1}=\frac{\sum_{1}^{9}\left(\mathrm{~h}_{i}-\mathrm{h}_{\text {min }}\right)}{\left(\mathrm{h}_{\text {max }}-\mathrm{h}_{\text {min }}\right) \cdot \mathrm{m}},
$$

where $R_{1}$ - retinal thickness criterion;

$\mathrm{h}_{i}$ - thickness of retinal sectors (in each sector of the macular zone), $\mu m$;

$\mathrm{h}_{\min }$ - retinal thickness in one of the scanning sectors of the macular zone, excluding the central and extreme sectors, $\mu m$;

$\mathrm{h}_{\max }-$ maximum retinal thickness of the macular zone, $\mu m$;

$\mathrm{m}-$ number of retinal scan sectors $(\mathrm{m}=9)$.

It is by the thickness of the retina that the doctor is able to determine the choice of treatment method (antiangiogenic therapy, laser retinal coagulation, a combination of these two methods).

The second device used to study retinal photosensitivity was the fundus microperimeter MAIA. The device operation is based on the method of neural networks. The device reflects the confocal image of the fundus on the touch screen, as well as provides a histogram, light sensitivity thresholds and color scale analysis of 37 points in the macular area [16].

A criterion assessing the effect of the patient's macular light sensitivity on the prognosis of diabetic retinopathy and diabetic macular edema in type 2 diabetes was developed. The formula is also based on the mathematical method of normalizing the studied indicator.

A criterion of the influence of photosensitivity, which characterizes the degree of change in the sensitivity thresholds of the macular zone of the retina taking into account the intensity of the light stimulus, is proposed:

$$
R_{2}=\frac{\sum_{1}^{36}\left(I_{i}-I_{\text {min }}\right)}{\left(I_{\max }-I_{\min }\right) \cdot 36},
$$

where $R_{2}$ - sensitivity criterion;

$I_{i}-$ light stimulus intensity, $d b$;

$I_{\text {min }}-$ minimum light stimulus intensity, $d b$;

$I_{\max }$ - maximum light stimulus intensity, $d b$;

36 - the number of points at which the light stimuli are determined.

Light sensitivity is the first to respond to changes in the retina caused by diabetes [6], [7], [8].

Studying the levels of biomarkers in serum (glycated hemoglobin HbA1c) and pathogenetic biomarkers in lacrimal fluid (vascular endothelial growth factor VEGF-A) makes it possible to objectively help to confirm the diagnosis of diabetic retinopathy. The advantage of studying biomarkers is the opportunity to determine the deviation of their levels in the patient from the norm, which allows to diagnose the disease, even if the ophthalmological equipment does not detect changes in the fundus.

It is proposed to take the level of glycated hemoglobin as the main serum blood biomarker describing the degree of compensation of diabetes mellitus. A criterion of the influence of glycated hemoglobin, which describes the nature of the relationship between the state of the morphological structures of the retina, corresponding to the severity of the 
volume of edema according to the value of the criterion and the degree of compensation of diabetes mellitus, is proposed:

$$
R_{3}=\frac{G}{G_{c r i}}
$$

where $R_{3}$ - glycated hemoglobin effect criterion;

$G$ - glycated hemoglobin level from the studied patient, \%;

$G_{c r i}$ - upper limit of glycated hemoglobin level, \%.

It is proposed to use the vascular endothelial factor (VEGF-A) as the main biomarker in the lacrimal fluid, which describes the pathogenetic mechanisms in the eye with the diabetic retinopathy. A criterion of the influence of the level of vascular endothelial growth factor, de-scribing the relationship between the state of the morphological structures of the retina and the level of vascular endothelial growth factor in the lacrimal fluid, is proposed:

$$
R_{4}=\frac{V E G F}{V E G F_{\max }},
$$

where $R_{4}$ - criterion of the influence of the vascular endothelial growth factor level;

$V E G F$ - level of vascular endothelial growth factor in the lacrimal fluid from the studied patient, $\mathrm{pg} / \mathrm{ml}$;

$V E G F_{\max }$ - maximum level of endothelial growth factor, $\mathrm{pg} / \mathrm{ml}$.

The developing formulas (1) - (4) are based on the mathematical method of normalizing the studying value. In the diagnosis of the diabetic retinopathy, it is proposed that all digital data can be divided into 4 factors which affect the diagnosis and prognosis of the disease ( $R_{1}$ - the influence of retinal thickness; $R_{2}$ - the influence of sensitivity; $R_{3}-$ the influence of the level of glycated hemoglobin; $R_{4}$ - the influence of the level of vascular endothelial growth factor).

All calculated criteria are combined in one integral criterion of progression of the disease:

$$
R_{\text {general }}=R_{1} \cdot R_{2} \cdot R_{3} \cdot R_{4},
$$

where $R_{\text {general }}$ - integral criterion of progression of the disease.

The boundary value table for the prognosis of diseases (table 1) was developed on the basis of statistical data processing.

Table 1. The prognosis of the disease depending on the criterion $R$

\begin{tabular}{|c|c|c|}
\hline$R_{\text {general }}$ & Disease diagnosis & Disease prognosis \\
\hline$R_{\text {general }} \leq 0.07$ & $\begin{array}{c}\text { Non-proliferative stage of } \\
\text { diabetic retinopathy with } \\
\text { diabetic macular edema }\end{array}$ & $\begin{array}{c}\text { Low risk of disease } \\
\text { progression with a good } \\
\text { prognosis for vision }\end{array}$ \\
\hline $0.07<R_{\text {general }}<0.18$ & $\begin{array}{c}\text { Preproliferative stage of } \\
\text { diabetic retinopathy with } \\
\text { diabetic macular edema }\end{array}$ & $\begin{array}{c}\text { High risk of disease } \\
\text { progression with a bad } \\
\text { prognosis for vision }\end{array}$ \\
\hline$R_{\text {general }} \geq 0.18$ & $\begin{array}{c}\text { Proliferative stage of } \\
\text { diabetic retinopathy with } \\
\text { diabetic macular edema }\end{array}$ & $\begin{array}{c}\text { High risk of disease } \\
\text { progression with a bad } \\
\text { prognosis for vision }\end{array}$ \\
\hline
\end{tabular}




\section{Results and discussion}

Based on the presented mathematical model, a program on prediction of diabetic retinopathy progression and diabetic macular edema was developed. The program is intended for researchers and practical ophthalmologists. The calculation in patients with diabetes mellitus is based on the use of criteria for evaluating the progression of the disease, taking into account the digital data of the latest ophthalmological equipment: optical coherence tomography, fundusmicroperimeter, as well as laboratory data: the level of vascular endothelial growth factor in the tear and glycated hemoglobin in the blood. The program allows to enter the initial data within two or three minutes and obtain the result. The result of the program calculation is a prognosis of the course of the disease.

To debug the program, the data from a patient's clinical example is used. To make a prognosis of the disease progression, the optical coherence tomography data, fundusmicroperimetry data, the level of glycated hemoglobin in the blood serum and the level of vascular endothelial growth factor in the lacrimal fluid must be entered into the program. A photoregistration of the fundus and a linear section of the retina by OCT in patient M, 63 years old with the diagnosis of diabetic retinopathy with diabetic macular edema is presented. (fig. 1).
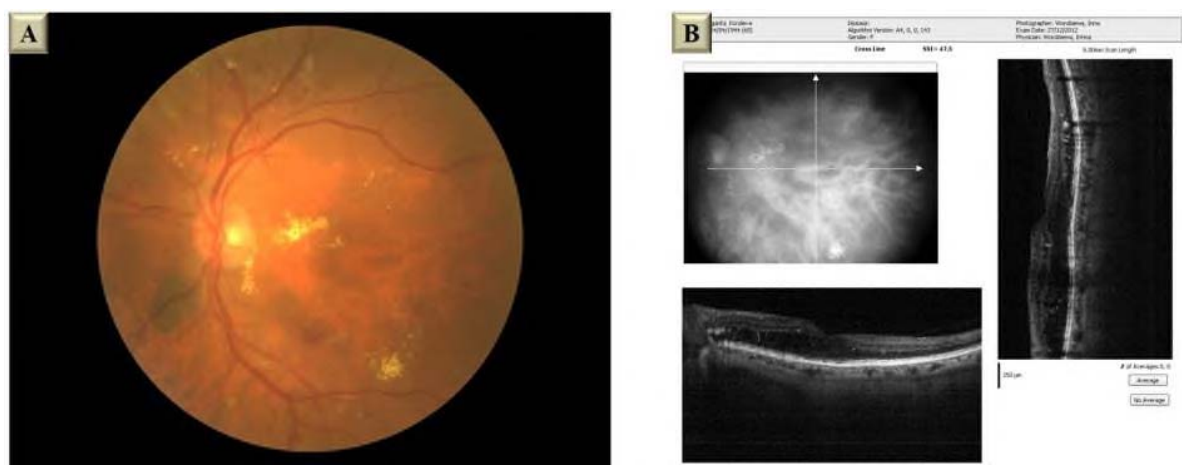

Fig. 1. Patient M, 63 years old with the diagnosis: diabetic retinopathy with diabetic macular edema (A - photoregistration of the fundus OS; B-OCT linear section of the retina OS)

The patient examination data are presented by an optical coherent tomograph (table 2, fig. 2), by the MAIA fundus microperimeter (table 3, fig. 3).

Table 2. Retinal thickness of the examined patient in the macula (fovea, parafovea, perifovea) according to the optical coherence tomograph data

\begin{tabular}{|c|c|c|}
\hline \multicolumn{2}{|c|}{ Section } & Thick, $\mu m$ \\
\hline \multicolumn{2}{|c|}{ Fovea centralis } & 419 \\
\hline \multirow{4}{*}{ ParaFovea } & Tempo & 363 \\
\hline & Superior & 361 \\
\hline & Nasal & 461 \\
\hline & Inferior & 488 \\
\hline \multirow{4}{*}{ PeriFovea } & Tempo & 294 \\
\hline & Superior & 326 \\
\hline & Nasal & 423 \\
\hline & Inferior & 444 \\
\hline
\end{tabular}


Table 3. Retinal light stimulus of the macula according to the MAIA fundus microperimeter data

\begin{tabular}{|c|c|}
\hline $\begin{array}{c}\text { Intensity of light stimulus, } \\
d b\end{array}$ & $\begin{array}{c}\text { The number of points with a } \\
\text { particular intensity }\end{array}$ \\
\hline 8 & 2 \\
\hline 10 & 2 \\
\hline 12 & 2 \\
\hline 14 & 10 \\
\hline 16 & 6 \\
\hline 18 & 12 \\
\hline 20 & 1 \\
\hline 22 & 1 \\
\hline 24 & 1 \\
\hline
\end{tabular}

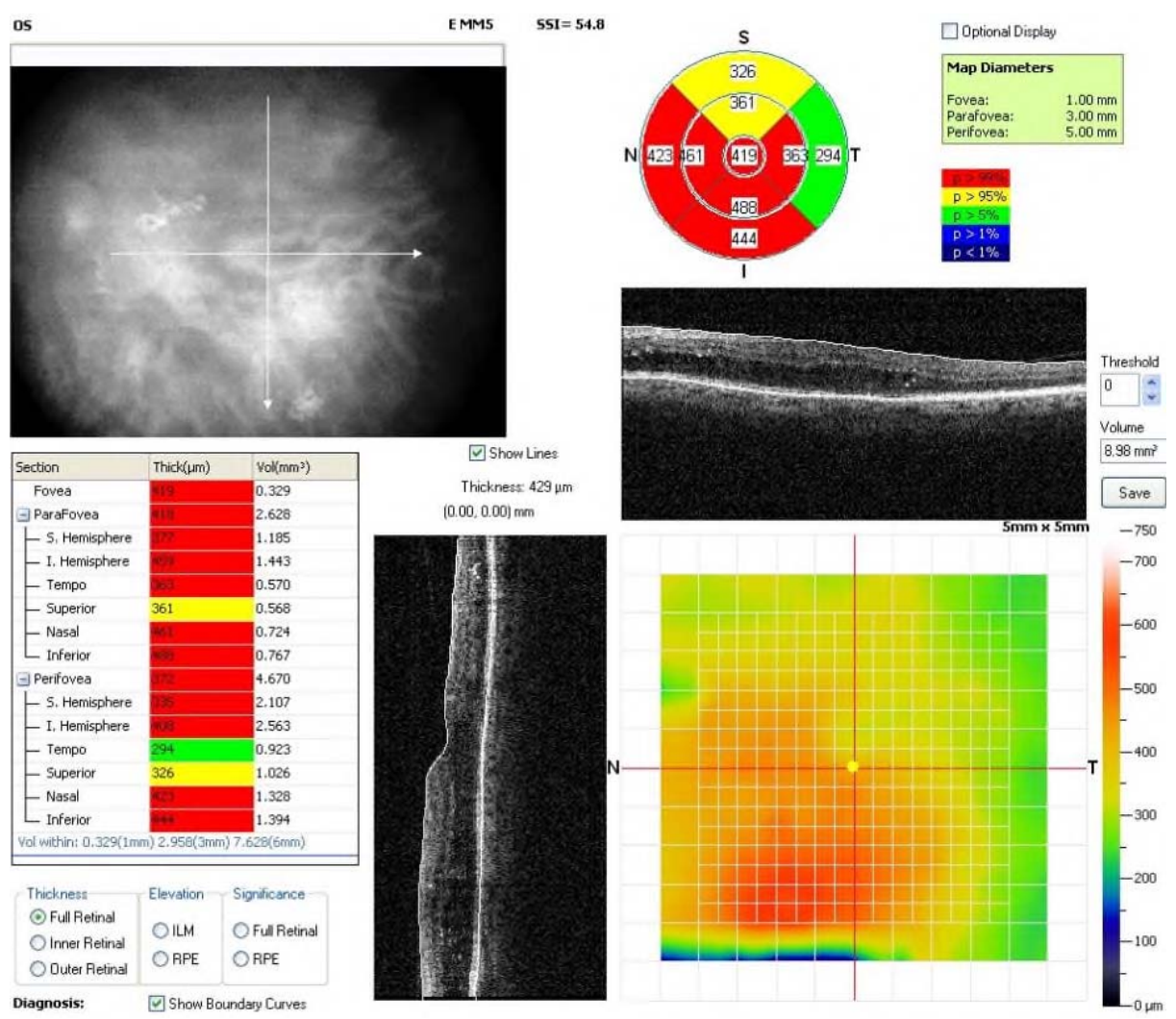

Fig. 2. Patient M, 63 years old with the diagnosis: diabetic retinopathy with diabetic macular edema. OCT OS: the thickness of the retina (Fovea, ParaFovea, PeriFovea)

Laboratory data is presented for determining the level of the biomarkers for the patient in blood serum and lacrimal fluid (table. 4).

Table 4. Laboratory data for determining the level of biomarkers for the patient in blood serum and lacrimal fluid

\begin{tabular}{|c|c|}
\hline $\begin{array}{c}\text { Level of serum glycated } \\
\text { hemoglobin, \% }\end{array}$ & $\begin{array}{c}\text { Level of vascular } \\
\text { endothelial growth factor } \\
\text { (VEGF-A) in the lacrimal } \\
\text { fluid, } \mathrm{pg} / \mathrm{ml}\end{array}$ \\
\hline 9.1 & 1400 \\
\hline
\end{tabular}


The result of the program calculation is the determination of the value of the integral criterion of progression $R_{\text {general }}=0.03$ and the automatic setting of the disease prognosis. The prognosis for the given patient is "low risk of disease progression with a favorable prognosis for vision" (table 1).

The risk of disease progression set in automatic mode according to the proposed mathematical model coincides with the doctor's conclusion.

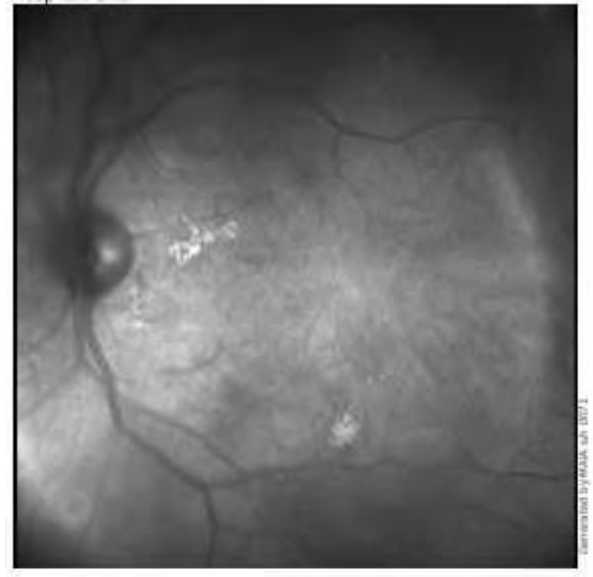

SENSITIVITY MAP

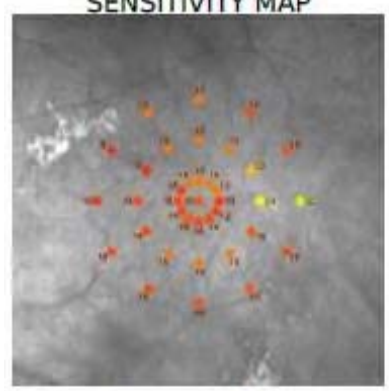

FIXATION PLOT

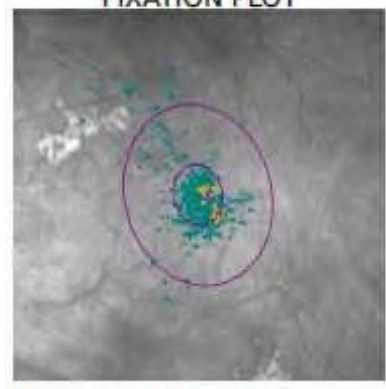

Bivariate Contour Ellipse Area:

$63 \%$ BCEA: $12^{\circ} \times 15^{\circ}$ Area $=5.7^{* 2}$, angle $=14.6^{\circ}$ $95 \%$ BCEA: $3.6^{\circ} \times 4.5^{\circ}$, Area $=51.2^{* 2}$, angle $=14.6^{\circ}$
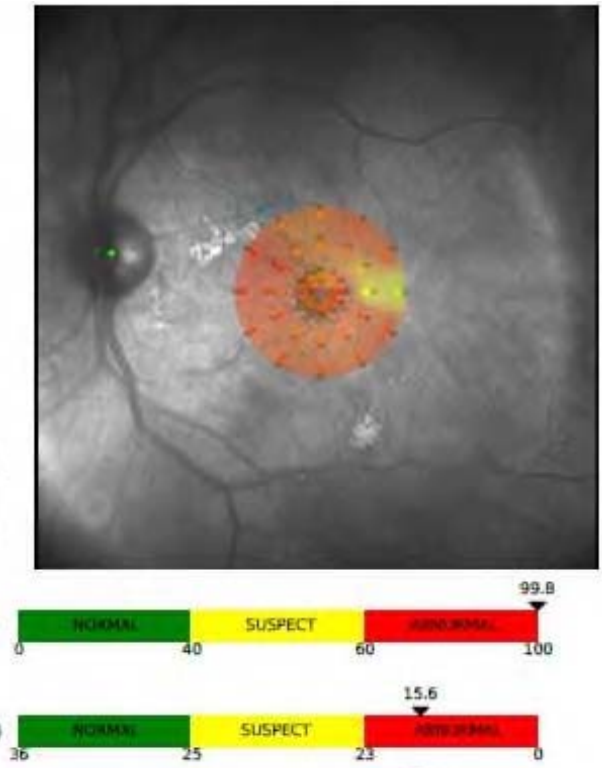

this exam

nermal populatian

Histogram of Threshald Frequencies
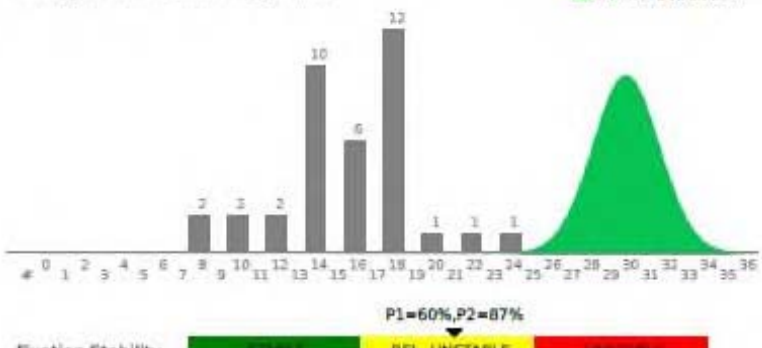

hel ungtagle

Fbation Graph

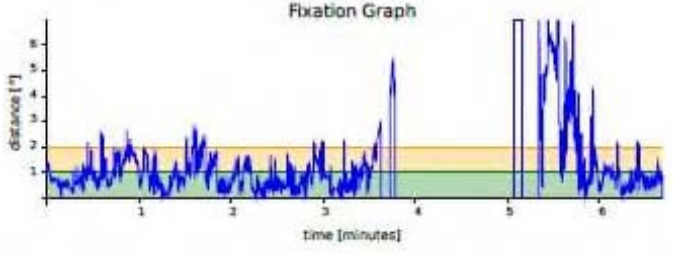

Fig. 3. Patient M, 63 years old with the diagnosis: diabetic retinopathy with diabetic macular edema. Macular photosensitivity by fundusmicroperimetry MAIA, histogram, 37 analyzed points of the macular zone, fixation point. 


\section{Conclusions}

A mathematical model that enables us to analyze a large amount of digital material for systematization of data from the optical coherence tomograph and fundusmicroperimeter MAIA is proposed.

A mathematical model for predicting diabetic retinopathy analyzes objective morphological data from an optical coherence tomograph, objective functional indicators of macular photosensitivity based on fundusmicroperimeter data, and objective indicators of biomarker levels in lacrimal fluid and serum.

The construction principle of the model is based on a mathematical method of normalizing the studied indicator taking into account: retinal thickness, macular photosensitivity, levels of biomarkers in the lacrimal fluid and blood serum.

The proposed prognosis of diabetic retinopathy with the help of the mathematical model gives the opportunity to reach a personalized approach to the patient, taking into account the complex of his individual indicators, which are calculated using formulas.

The proposed program enables the doctor to analyze the results of the examination and to quickly get a forecast of the risk of developing disease.

The created effective mathematical model gives the opportunity to assist in the formation of a decision support system, to reach an interdisciplinary level of research using precise mathematics, digital technologies and methods of medical engineering to conduct a quantitative and qualitative assessment of the fundus structures.

The criteria obtained by the formulas are comparable with the results of clinical diagnosis and evaluation of fundus structures.

\section{References}

1. V.G. Gagarin, V.K. Akhmetov and K.P. Zubarev position control of maximum wetting plane for building walls with foam polystyrene heat insulator, IOP Conference Series: Materials Science and Engineering, 753, L 022045 (2020)

2. V.G. Gagarin, V.K. Akhmetov and K.P. Zubarev Graphical method for determination of maximum wetting plane position in enclosing structures of buildings, IOP Conference Series: Materials Science and Engineering (International science and technology conference "FarEastCon-2019"), 753, L 022045 (2020)

3. V.G. Gagarin, V.K. Akhmetov and K.P. Zubarev The moisture regime calculation of single-layer enclosing structures on the basis of the discrete-continuum method application, IOP Conference Series: Materials Science and Engineering, 456, L 012105 (2018)

4. V.G. Gagarin, V.K. Akhmetov and K.P. Zubarev Assessment of enclosing structure moisture regime using moisture potential theory, MATEC Web of Conferences, 193, L $03053(2018)$

5. V.G. Gagarin, V.K. Akhmetov and K.P. Zubarev Moisture behavior calculation of single-layer enclosing structure by means of discrete-continuous method, MATEC Web of Conferences, 170, L 03014 (2018)

6. I.V. Vorobyeva, L.K. Moshetova and E.V. Shcherbakova Therapy results in patients with primary open angle glaucoma and diabetic retinopathy, Ophthalmology in Russia, 14, L 1, 40-46 (2017)

7. I.V. Vorobyeva Long-term results of treatment of patients with non-proliferative diabetic retinopathy angioprotectors, Ophthalmology in Russia, 14, L 1, 67-77 (2017) 
8. I.V. Vorobyeva Monitoring of separate pathogenetically significant biochemical markers in lacrimal fluid, ophthalmological parameters with combined pathology of diabetic retinopathy and age-related macular degeneration on the background angioprotective and antioxidant therapy, Ophthalmology in Russia, 15, L 2, 189-99 (2018)

9. S. Stolte and RG. Fang A survey on medical image analysis in diabetic retinopathy, Medical image analysis, 64, L 101742, 1-75 (2020)

10. NQ. Xie, Y. Tan, S. Liu, YN. Xie, SS. Shuai, W. Wang, and WY. Huang Macular vessel density in diabetes and diabetic retinopathy with swept-source optical coherence tomography angiography, Graefes archive for clinical and experimental ophthalmology (2020)

11. C. Sun, HS. Zhang, JJ. Jiang, YX. Li, C. Nie, JW. Gu, L. Luo and ZJ. Wang Angiogenic and inflammatory biomarker levels in aqueous humor and vitreous of neovascular glaucoma and proliferative diabetic retinopathy, International ophthalmology, 40, L 2, 467-475 (2020)

12. YD. Zhang, XM. Zhang, Z. Gao, ZG. Yuan, T. Ma, GY. Li and XH. Gao Correlations of the severity of type 2 diabetic retinopathy with vascular endothelial growth factor, inflammatory factors and oxidative stress in serum and aqueous humor, Journal of biomaterials and tissue engineering, 10, L 3, 424-428 (2020)

13. X. Li, X. Gu, T. Boyce, M. Zheng, A. Reagan, H. Qi, N. Mandal, A. Cohen, M. Callegan, D. Carr and M. Elliott Caveolin-1 increases proinflammatory chemoattractants and blood-retinal barrier breakdown but decreases leukocyte recruitment in inflammation, Investigative ophthalmology and visual science, 55, L 10, 6224-6234, (2020)

14. E. Stefansson, Y. Chan, T. Bek, S. Hardarson, D. Wong and D. Wilson, Laws of physics help explain capillary non-perfusion in diabetic retinopathy, Eye, 32, L 2, 210 212 (2018)

15. S. Lund, T. Aspelund, P. Kirby, G. Russell, S. Einarsson, O. Palsson and E. Stefansson individualised risk assessment for diabetic retinopathy and optimisation of screening intervals: a scientific approach to reducing healthcare Costs, Medical image analysis, 100, L 5, 683-687 (2016)

16. S.N. Markowitz and S.V. Reyes microperimetry and clinical practice: an evidencebased review, Canadian journal of ophthalmology, 48, L 5, 350-357, (2013) 